\title{
DPT/BCG/Measles/Serratia/Pneumococcus Vaccine
}

National Cancer Institute

\section{Source}

National Cancer Institute. DPT/BCG/Measles/Serratia/Pneumococcus Vaccine. NCI

Thesaurus. Code C120213.

A proprietary lipid emulsion containing five vaccines: diphtheria, pertussis, tetanus (DPT), Bacille Calmette-Guerin (BCG), measles, Serratia marcescens and pneumococcal, with potential immunostimulating activity. Subcutaneous administration of the $\mathrm{DPT} / \mathrm{BCG} / \mathrm{measles/Serratia/pneumococcus} \mathrm{vaccine} \mathrm{activates} \mathrm{the} \mathrm{immune} \mathrm{system} \mathrm{and}$ may both abrogate tumor-induced immune tolerance and induce an antitumor immune response, which may eradicate the tumor. 